\title{
Pooled cohort equations heart failure risk score predicts cardiovascular disease and all-cause mortality in a nationally representative sample of US adults
}

Alexander C. Razavi ${ }^{1,2^{*}}$, Kaitlin S. Potts ${ }^{1}$, Tanika N. Kelly ${ }^{1}$, Jiang He ${ }^{1,2}$, Camilo Fernandez ${ }^{1,2}$, Marie Krousel-Wood ${ }^{1,2}$, Amanda H. Anderson', Joshua Bundy ${ }^{1}$, Seamus P. Whelton ${ }^{3}$, Roger S. Blumenthal ${ }^{3}$, Donald Lloyd-Jones ${ }^{4}$ and Lydia A. Bazzano ${ }^{1,2}$

\begin{abstract}
Background: Heart failure (HF) represents an accumulated burden of systemic vascular damage and is the fastest growing form of cardiovascular disease (CVD). Due to increasing HF-attributable mortality rates, we sought to assess the association of the new 2019 Pooled Cohort equations to Prevent Heart Failure (PCP-HF) risk score with CVD and all-cause mortality.

Methods: We linked data for 6333 black and white men and women aged 40-79years, whom underwent electrocardiographic examination from the Third National Health and Nutrition Exam Survey, to National Death Index record matches. Sex- and race-specific PCP-HF risk scores were calculated using data on age, smoking, body mass index, systolic blood pressure, total cholesterol, HDL-cholesterol, fasting blood glucose, QRS complex duration, and antihypertensive and/or glucose-lowering medications. Cox regression estimated hazard ratios for the association of the PCP-HF risk score with CVD and all-cause mortality.

Results: Individuals were on average 54.9 years old (51.7\% women, $25.4 \%$ black) and the median 10-year HF risk was 1.6\% $(\mathrm{Q} 1=0.5, \mathrm{Q} 3=4.8)$. There were 3178 deaths, 1116 from CVD, over a median follow-up time of 22.3 years. Black women had a higher 10-year HF risk compared to white women (2.1\% vs. 1.1\%; $p<0.01)$, while no significant difference was observed in predicted HF risk between black men and white men (2.3\% vs. $2.1 \%, p=0.16)$. A twofold higher PCP-HF risk score was associated with a significant $58 \%(\mathrm{HR}=1.58 ; 95 \% \mathrm{Cl}, 1.48-1.70 ; p<0.0001)$ and $38 \%(\mathrm{HR}=1.38 ; 95 \% \mathrm{Cl}, 1.32-1.46 ; \mathrm{p}<0.0001)$ greater risk of CVD and all-cause mortality, respectively.

\footnotetext{
*Correspondence: arazavi@tulane.edu

'Department of Epidemiology, Tulane University School of Public Health and

Tropical Medicine, 1440 Canal Street, Suite 2000, New Orleans, Louisiana

70112, USA

${ }^{2}$ Department of Medicine, Tulane University School of Medicine, New

Orleans, Louisiana, USA

Full list of author information is available at the end of the article
}

C The Author(s). 2020 Open Access This article is licensed under a Creative Commons Attribution 4.0 International License, which permits use, sharing, adaptation, distribution and reproduction in any medium or format, as long as you give appropriate credit to the original author(s) and the source, provide a link to the Creative Commons licence, and indicate if changes were made. The images or other third party material in this article are included in the article's Creative Commons licence, unless indicated otherwise in a credit line to the material. If material is not included in the article's Creative Commons licence and your intended use is not permitted by statutory regulation or exceeds the permitted use, you will need to obtain permission directly from the copyright holder. To view a copy of this licence, visit http://creativecommons.org/licenses/by/4.0/. The Creative Commons Public Domain Dedication waiver (http://creativecommons.org/publicdomain/zero/1.0/) applies to the data made available in this article, unless otherwise stated in a credit line to the data. 
(Continued from previous page)

Conclusion: The PCP-HF risk score predicts CVD and all-cause mortality, in addition to the 10-year risk of incident HF among white and black men and women. These results underline the expanded utility of the PCP-HF risk score and suggest that its implementation in the clinical and population health settings may improve primary CVD prevention in the United States.

Keywords: Heart failure, Risk, Cardiovascular diseases, Mortality, Primary prevention, NHANES, Social determinants of health, Epidemiology, Blood pressure, Electrocardiography

\section{Background}

Heart failure (HF) is the fastest growing form of cardiovascular disease (CVD) nationally and globally, as its prevalence is projected to increase by $46 \%$ over the next decade in the United States [1-3]. The HF-attributable mortality rate has increased by over $20 \%$ since 2011 [4] and the median survival time after an initial HF diagnosis is as low as 2.1 years [5], differing by race, ethnicity, and socioeconomic status [6]. Because HF is primarily a non-reversible disease, primary prevention is a key strategy to reducing population morbidity and mortality. Similar to other forms of CVD, HF may be largely preventable through lifestyle approaches and modification of common risk factors, including hypertension, dyslipidemia, obesity, and type II diabetes [7].

In an effort to improve HF risk stratification and prevention, the Pooled Cohort equations to Prevent HF (PCP-HF) risk score was developed and validated in 2019 using combined individual-level data from five diverse population-based cohorts to estimate the 10-year risk for incident HF [8]. Though a variety of HF risk scores exist [9-12], the PCP-HF contains parameters that are easily obtained in a primary care setting, and was developed with information from younger adults as well black men and women, in contrast to current risk prediction tools.

Data from both observational studies [13] and randomized controlled trials [14] indicate that HF is a heterogeneous disease, and suggests that further effort must be directed more upstream on the causal disease pathway, prior to clinical HF manifestation. While the PCP-HF risk score has successfully been validated for HF risk prediction in black and white adults across cohort studies [8], it is not known whether the PCPHF risk score predicts future CVD- and/or all-cause mortality. Likewise, differences in the estimated 10year risk for incident HF according to level of income, education, marital status, and living environment remain ill-defined. Information on the social determinants that may help characterize different patterns of HF in vulnerable populations may inform early preventive action against HF onset, and identify epidemiologic and clinically pertinent differences in predicted HF risk among key sociodemographic populations. To our knowledge, this is the first investigation of the predictive and discriminative ability of the PCP-HF risk score in the general United States population using the National Health and Nutrition Examination Survey (NHANES). We estimated the 10-year risk for incident HF and assessed the association of the PCP-HF risk score with CVD- and allcause mortality in black and white women and men living in the United States.

\section{Methods}

The NHANES uses a complex, stratified, multistage probability cluster sampling design to select a representative sample of noninstitutionalized citizens living in the United States. The purpose of these surveys is to describe the epidemiology of multiple conditions and health status of Americans. The baseline examinations for NHANES III occurred from 1988 to 1994 and detailed methods regarding data collection have been previously described [15]. National Death Index searches, made available through public datasets at the National Center for Health Statistics, were linked to NHANES III participants and provided mortality follow-up data [16]. A total of 8561 men and women aged 40-79 years had available electrocardiogram data with measures of QRS complex duration. After excluding individuals missing data necessary to derive PCP-HF risk scores $(n=1775)$, and individuals with self-reported HF $(n=453), 6333$ persons remained in the analysis. Among these 6333 persons were 2302 white men, 2421 white women, 754 black men, and 856 black women.

Standardized household interviews were used to collect information on sociodemographic variables, including sex, race, medical/medication history, income and education level, marital status, and area of residence. Physical examinations were performed at a mobile examination center. Blood pressure was measured in triplicate using a mercury sphygmomanometer by trained physicians after study participants had undergone 5 min of rest [17]. A twelve-lead electrocardiogram was performed with study participants still, supine and breathing normally. Electrocardiogram data and tracings were uploaded to and stored on a Marquette MAC 12 unit for accurate analysis [18]. An electronic digital scale 
and steel tape measure were used to measure weight and height respectively [19]. Total serum cholesterol was measured enzymatically, while HDL-cholesterol was measured after precipitation of other lipoproteins with a polyanion/divalent cation mixture. Glucose was measured enzymatically in plasma in the fasted state via the hexokinase method [20].

Sex- and race-specific 10-year PCP-HF risk scores were calculated via equations derived by Khan et al. [8]. Individual coefficient values were calculated for each sex and race group using data on age, treated or untreated systolic blood pressure, smoking status, treated or untreated fasting glucose, total cholesterol, HDLcholesterol, body mass index, and QRS complex duration. Coefficient values were summed to generate an individual coefficient value for each study participant. Both the individual coefficient values and mean coefficient values were then used to estimate the 10-year risk of a HF event through the following formal equation as

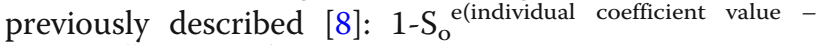
mean coefficient value). Further explanation of risk score components and calculations are provided in Supplemental Table 1.

CVD mortality and all-cause mortality were defined as the primary and secondary outcome, respectively, for this observational study. CVD mortality was defined using the International Classification of Diseases-10th Revision (ICD-10) codes, I00-I09, I11, I13, I20-I51, I60I69 [21].

We described the sample according to United States population weights, using means for normally distributed continuous variables, medians for non-normally distributed continuous variables, and percentages for categorical variables in the total sample. Weighted standard errors, interquartile ranges, and ranges were presented as measures of dispersion when appropriate. Normality of continuous variables was assessed via the Kolmogorov-Smirnov test. The PCP-HF risk score was transformed to the $\log _{2}$ scale to normalize its distribution prior to multivariable analysis. This method of transformation was chosen for interpretation purposes, as a one unit increase on the $\log _{2}$ scale corresponds to a doubling, or two-fold increase in the transformed variable. The Student's t-test and Wilcoxon signed-rank test were used to assess differences in normally and nonnormally distributed continuous variables, respectively. Differences between categorical variables were evaluated using Pearson's chi-square test. Survival curves by quartile of PCP-HF risk score were plotted and compared using the log-rank test to assess differences in CVD and all-cause mortality among risk quartiles. We conducted race- and sex-stratified analyses when appropriate.

Crude CVD-attributed death rates were calculated per PCP-HF risk score quartile as the number of CVD deaths divided by the sum of follow-up years per 1000 person-years for each five-year age strata. Age-adjusted CVD mortality rates per quartile of PCP-HF risk score were calculated by standardizing to the age-distribution of the total sample. Standard errors of the age-adjusted rates were estimated by dividing the age-adjusted rate by the square-root of the number of events [22]. These were used to calculate 95\% confidence intervals (CIs) and compare age-adjusted CVD mortality rates across PCP-HF risk score quartile. Trend in age-adjusted mortality across PCP-HF risk score quartiles was tested using the median quartile value for each individual observation.

The association of the PCP-HF risk score with CVD and all-cause mortality was assessed using Cox proportional hazards regression models. The proportional hazards assumptions were found to be appropriate. Potential confounding variables were chosen a priori based on established associations with HF and CVD mortality [23]. We estimated the association between the PCP-HF and death due to CVD and all-causes using three models: 1) unadjusted; 2) adjusted for age, sex, and race; 3 ) adjusted for variables in model 2 plus previous history of myocardial infarction, annual income level (below $\$ 25,000$ versus $\$ 25,000-\$ 50,000$ versus more than $\$ 50,000$ ), education status (high school education or lower versus post high school education), living environment (urban versus non-urban), and living status (living alone versus living with a partner). Discrimination ability of the models to differentiate between events and non-events was evaluated using $\mathrm{C}$-statistics. C-statistics were computed and presented as a measure of discrimination in the context of prediction accuracy of the model, as previously described $[24,25]$. Statistical analyses were performed using R and SAS 9.3 (SAS Institute, Cary, NC). Sample weights and complex survey design effect were accounted for in all analyses ( $R$ package "survey"; SAS procedures surveymeans, surveyfreq, surveyphreg, surveyreg, and surveylogistic). All hypothesis tests were 2 -sided. We used an alpha threshold of 0.05 for detecting differences in descriptive statistics and a Bonferroni-corrected alpha threshold of $0.025(0.05 / 2)$ for proportional hazard regression models (primary outcome $=$ CVD mortality; secondary outcome $=$ all-cause mortality).

\section{Results}

Table 1 presents baseline characteristics and mortality data according to United States population sampling weights for the 6333 NHANES participants (mean age: 54.9 years; 3277 women; 1610 black) included in the analyses. The study sample, on average, was overweight $\left(\mathrm{BMI}=27.4+0.1 \mathrm{~kg} / \mathrm{m}^{2}\right)$ and had elevated systolic blood pressure $(129.7+0.4 \mathrm{mmHg})$ and cholesterol (total 
Table 1 Characteristics of 6333 Black and White NHANES III Participants Without Heart Failure

\begin{tabular}{|c|c|c|c|c|c|c|}
\hline Variable*† & $\begin{array}{l}\text { All } \\
(\boldsymbol{n}=6333)\end{array}$ & $\begin{array}{l}\text { PCP-HF Q1 } \\
(\boldsymbol{n}=1583)\end{array}$ & $\begin{array}{l}\text { PCP-HF Q2 } \\
(n=1583)\end{array}$ & $\begin{array}{l}\text { PCP-HF Q3 } \\
(\boldsymbol{n}=1584)\end{array}$ & $\begin{array}{l}\text { PCP-HF Q4 } \\
(n=1583)\end{array}$ & $\boldsymbol{P}$-value for Trend \\
\hline \multicolumn{7}{|l|}{ Sociodemographic \& Lifestyle } \\
\hline Age, years, mean & $54.9(0.3)$ & $44.9(0.2)$ & $53.1(0.3)$ & $62.4(0.4)$ & $68.4(0.4)$ & $<0.0001$ \\
\hline Female, \% & $52.3(0.7)$ & $62.6(1.2)$ & $49.9(1.6)$ & $48.8(1.8)$ & $39.6(1.8)$ & $<0.0001$ \\
\hline Black, \% & $9.2(0.6)$ & $5.8(0.5)$ & $11.6(0.8)$ & $11.9(0.9)$ & $9.1(1.0)$ & 0.08 \\
\hline Current Smoking, \% & $24.3(1.0)$ & $18.0(1.6)$ & $32.1(2.0)$ & $23.9(1.5)$ & $25.3(2.0)$ & 0.19 \\
\hline Living Environment, \% & & & & & & 0.15 \\
\hline Urban & $46.1(4.6)$ & $47.4(5.1)$ & $47.2(4.9)$ & $45.3(4.4)$ & $42.9(4.8)$ & \\
\hline Non-Urban & $53.9(4.6)$ & $52.6(5.1)$ & $52.8(4.9)$ & $54.7(4.4)$ & $57.1(4.8)$ & \\
\hline Living Status, \% & & & & & & $<0.001$ \\
\hline Living Alone & $26.7(0.9)$ & $23.3(1.7)$ & $25.0(1.9)$ & $29.7(1.7)$ & $32.6(1.7)$ & \\
\hline Living with Partner & $73.3(0.9)$ & $76.7(1.7)$ & $75.0(1.9)$ & $70.3(1.7)$ & $67.4(1.7)$ & \\
\hline Educational Attainment, \% & & & & & & $<0.0001$ \\
\hline High-School Education or Below & $60.7(1.6)$ & $47.1(2.3)$ & $61.9(2.2)$ & $71.1(1.9)$ & $73.6(2.0)$ & \\
\hline Post High-School Education & $39.3(1.6)$ & $52.9(2.3)$ & $38.1(2.2)$ & $28.9(1.9)$ & $26.4(2.0)$ & \\
\hline Household Income Level, \% & & & & & & $<0.0001$ \\
\hline Below $\$ 25,000$ & $40.8(1.5)$ & $25.5(1.7)$ & $37.5(2.1)$ & $52.4(2.0)$ & $62.6(2.2)$ & \\
\hline$\$ 25,000-\$ 50,000$ & $32.1(1.1)$ & $34.6(2.0)$ & $35.2(1.6)$ & $30.2(1.9)$ & $24.5(2.1)$ & \\
\hline Above $\$ 50,000$ & $27.1(1.6)$ & $39.9(2.5)$ & $27.3(2.0)$ & $17.4(1.6)$ & $12.9(1.8)$ & \\
\hline \multicolumn{7}{|l|}{ Cardiovascular } \\
\hline 10-Year HF Risk, median (min, max) & $1.6(0.0,82.7)$ & $0.3(0.0,0.8)$ & $1.5(0.8,2.7)$ & $4.2(2.7,6.7)$ & $10.8(6.7,82.7)$ & $<0.0001$ \\
\hline QRS Duration, milliseconds, mean & $98.3(0.5)$ & $96.3(0.6)$ & $97.5(0.7)$ & $98.5(0.6)$ & $103.2(0.8)$ & $<0.0001$ \\
\hline Systolic Blood Pressure, mmHg, mean & $129.7(0.4)$ & $118.7(0.6)$ & $128.5(0.6)$ & $136.8(0.6)$ & $145.1(0.8)$ & $<0.0001$ \\
\hline Previous Myocardial Infarction, \% & $3.8(0.3)$ & $0.8(0.4)$ & $1.9(0.5)$ & $5.5(0.9)$ & $10.2(1.0)$ & $<0.0001$ \\
\hline Antihypertensive Medication, $\%$ & $18.8(0.7)$ & $2.3(0.6)$ & $12.8(1.1)$ & $24.9(1.5)$ & $53.5(1.8)$ & $<0.0001$ \\
\hline \multicolumn{7}{|l|}{ Metabolic } \\
\hline Body Mass Index, $\mathrm{kg} / \mathrm{m}^{2}$, mean & $27.4(0.1)$ & $25.5(0.1)$ & $28.0(0.3)$ & $28.3(0.2)$ & $29.2(0.2)$ & $<0.0001$ \\
\hline Total Cholesterol, mg/dL, mean & $216.7(0.9)$ & $206.5(1.4)$ & $219.0(1.5)$ & $226.5(1.6)$ & $222.1(1.2)$ & $<0.0001$ \\
\hline HDL Cholesterol, mg/dL, mean & $50.7(0.5)$ & $53.7(0.6)$ & $50.4(0.7)$ & $50.0(0.6)$ & $45.8(0.7)$ & $<0.0001$ \\
\hline Fasting Blood Glucose, mg/dL, mean & $101.3(0.8)$ & $91.5(0.5)$ & $96.8(1.0)$ & $101.6(1.1)$ & $127.2(2.2)$ & $<0.0001$ \\
\hline Glucose-Lowering Medication, \% & $5.0(0.3)$ & $0.2(0.1)$ & $1.3(0.3)$ & $4.8(0.7)$ & $20.0(1.8)$ & $<0.0001$ \\
\hline \multicolumn{7}{|l|}{ Mortality } \\
\hline Cardiovascular Disease Mortality, \% & $13.8(0.6)$ & $2.2(0.4)$ & $6.9(0.8)$ & $23.4(1.4)$ & $36.1(1.5)$ & $<0.0001$ \\
\hline All-cause Mortality, \% & $41.7(1.3)$ & $11.3(1.2)$ & $31.7(1.7)$ & $65.5(1.9)$ & $88.8(1.1)$ & $<0.0001$ \\
\hline
\end{tabular}

* = percentages, means, medians, and measures of dispersion are estimated using U.S. population weights

$\dagger=$ measure of dispersion is standard error unless otherwise noted

$H D L$ high-density lipoprotein, Q1 quartile 1, Q2 quartile 3, Q3 quartile 3, Q4 quartile 4, PCP-HF Pooled Cohort equations to Prevent Heart Failure

cholesterol $=216.7+0.9 \mathrm{mg} / \mathrm{dL})$. A total of 3178 individuals died during a median follow-up time of 22.3 years, and 1116 deaths were attributed to CVD (Supplemental Table 2). There were significantly more men, individuals living alone, and individuals with low educational attainment per increasing PCP-HF risk score quartile. Additionally, we observed a higher proportion of CVDattributable and total deaths when comparing trends from the lowest to highest PCP-HF risk score quartiles.
The estimated median 10-year risk for incident $\mathrm{HF}$ was $1.6 \%$. Figure 1 compares the average 10 -year risk for incident HF by previous history of myocardial infarction and sociodemographic variables. Individuals selfreporting a previous myocardial infarction had a significantly higher predicted 10-year risk for HF compared with those who did not report a history of myocardial infarction $(6.4 \%$ vs. $1.5 \% ; p<0.0001)$. Black women, adults living alone, living in rural settings, and adults 


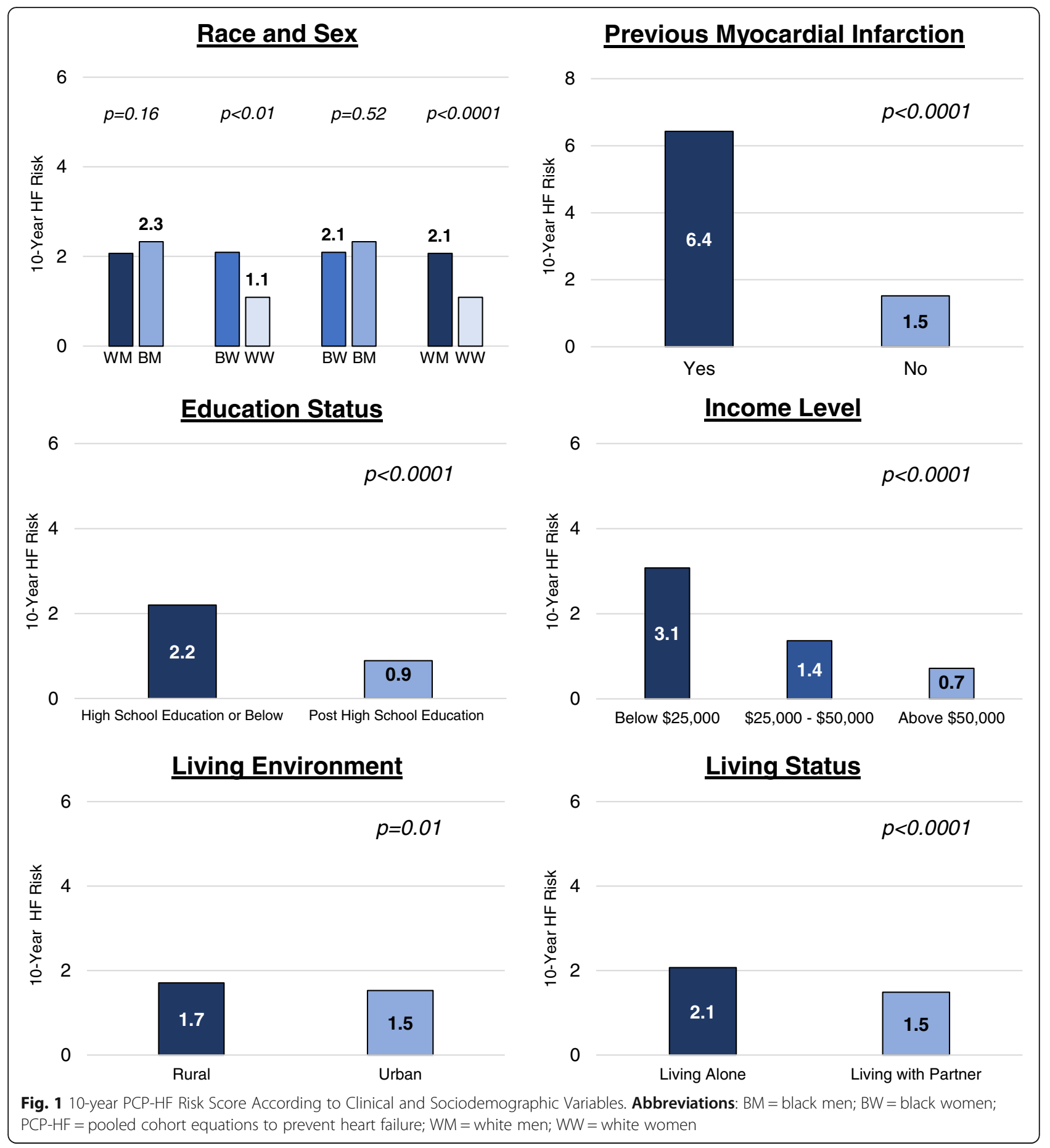

with a high school education or lower had significantly higher PCP-HF risk scores compared to white women, adults living with partners, adults living in urban environments, and post high school graduates, respectively. White men also had a higher 10-year HF risk compared to white women $(2.1 \%$ vs. $1.1 \%, \mathrm{p}<0.0001)$. Likewise, there were significant differences in HF risk according to annual income levels, with adults living in households with an annual income less than $\$ 25,000$ having the highest estimated 10-year risk for incident HF (3.1\%) among all income level strata.

Fig. 2 presents CVD and all-cause mortality survival probabilities stratified by PCP-HF risk score quartile over follow-up time. There were significant differences in survival among PCP-HF risk score quartiles $(p<$ 0.0001) for both outcomes, and there was a shorter 


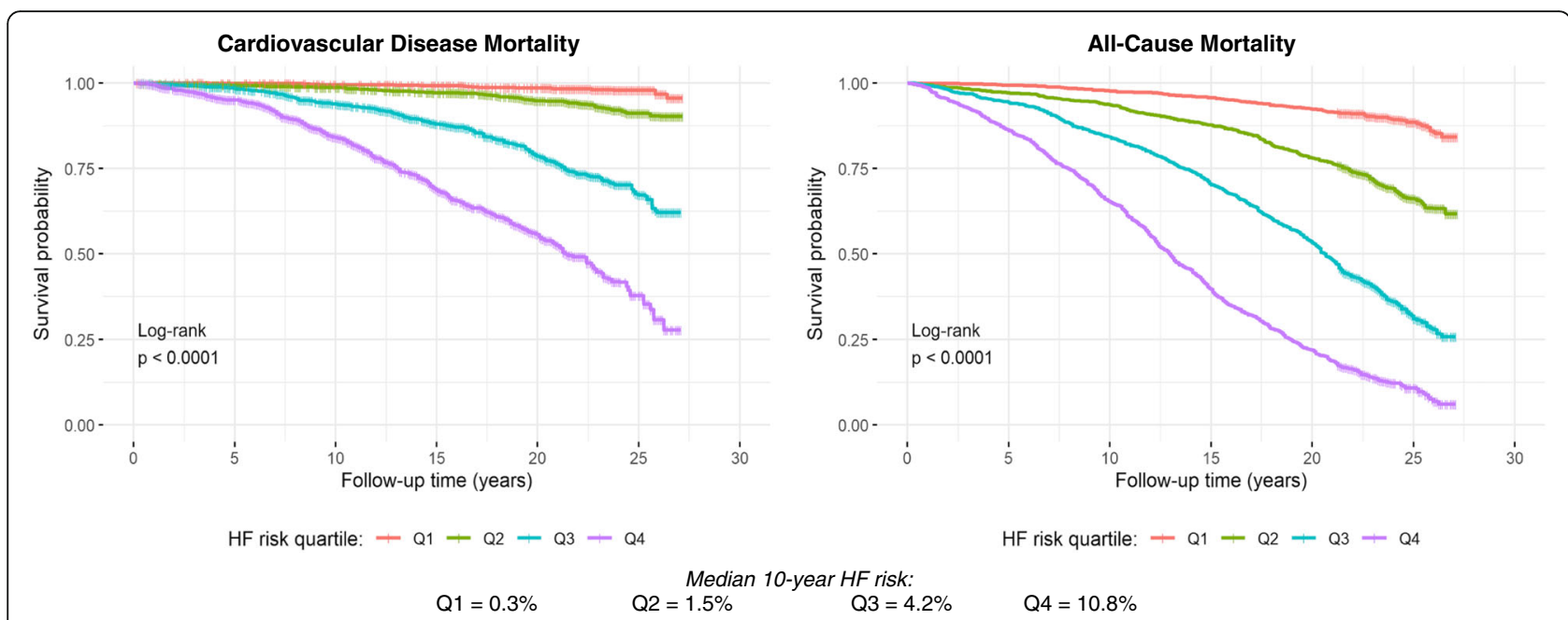

Fig. 2 Kaplan Meier Estimates of Cardiovascular Disease and All-Cause Survival Probability According to PCP-HF Risk Score Quartiles.

Abbreviations: $\mathrm{PCP}-\mathrm{HF}=$ pooled cohort equations to prevent heart failure

median survival time with each increasing PCP-HF risk score quartile. Among individuals with the highest 10year risk for incident HF (quartile 4), median all-cause and CVD survival was 12.6 and 21.3 years, respectively.

The association of the PCP-HF risk score with death due to CVD and all-causes is presented in Fig. 3 and Table 2. We observed a significant upward trend $(p<$ 0.0001) in age-adjusted all-cause and CVD mortality rates from the lowest to highest quartile of PCP-HF risk score (Fig. 3). The age-adjusted CVD mortality rate for quartile 4 was 31.3 events per 1000 person-years, more than 11 times greater than the CVD mortality rate for individuals in the lowest quartile (2.8 events per 1000 person-years). Likewise, there was an 8-fold higher allcause mortality rate when comparing the highest
(72.8 events per 1000 person-years) versus the lowest PCP-HF risk score quartile (8.6 events per 1000 personyears). After adjusting for sociodemographic variables and previous history of myocardial infarction, there was a 58 and $38 \%$ higher risk of death due to CVD (HR = $1.58,95 \% \mathrm{CI}=1.48,1.70 ; p<0.0001)$ and all-causes $(\mathrm{HR}=1.38,95 \% \mathrm{CI}=1.32,1.46 ; \mathrm{p}<0.0001)$, for every two-fold increase in the PCP-HF risk score (Table 2).

In unadjusted models, there was good discriminative ability of the PCP-HF risk score, and the risk score predicted CVD mortality $(C$-statistic $=0.795)$ to a better extent compared to all-cause mortality $(C$-statistic $=0.750)$. The addition of sociodemographic variables and history of myocardial infarction only modestly improved discriminative ability for both CVD $(\mathrm{C}$-statistic $=0.812)$ and

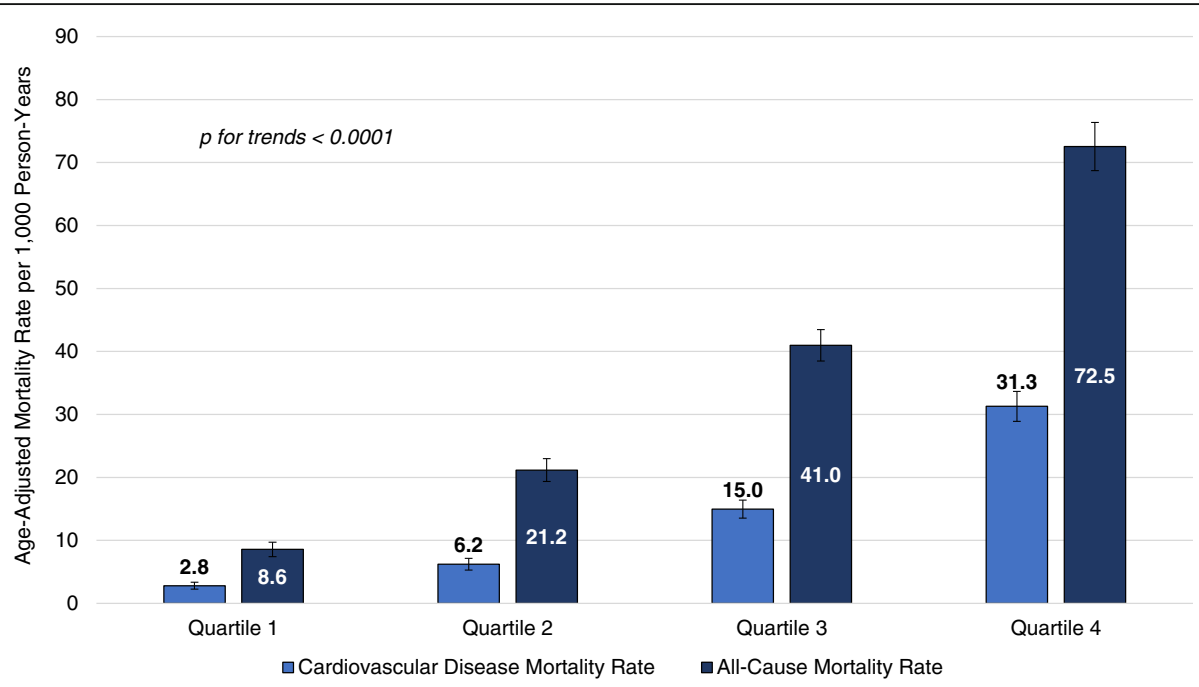

Fig. 3 Age-adjusted Cardiovascular Disease and All-Cause Mortality Rates According to PCP-HF Risk Score Quartiles. Abbreviations: PCP-HF = pooled cohort equations to prevent heart failure 
Table 2 Hazard of Cardiovascular Disease and All-Cause Mortality According to Continuous PCP-HF Risk Score

\begin{tabular}{|c|c|c|c|c|c|c|}
\hline \multirow[t]{2}{*}{ Variable } & \multicolumn{2}{|l|}{ Unadjusted } & \multicolumn{2}{|l|}{ Model $2^{*}$} & \multicolumn{2}{|l|}{ Model $3+$} \\
\hline & Hazard Ratio (95\% Cl) & $P$-value & Hazard Ratio $(95 \% \mathrm{Cl})$ & $\boldsymbol{P}$-value & Hazard Ratio (95\% Cl) & $\boldsymbol{P}$-value \\
\hline \multicolumn{7}{|c|}{ Cardiovascular Disease Mortality } \\
\hline PCP-HF Risk Score $\ddagger$ & $2.03(1.92,2.15)$ & $<0.0001$ & $1.64(1.54,1.75)$ & $<0.0001$ & $1.58(1.48,1.70)$ & $<0.0001$ \\
\hline (per doubling) & C-statistic $=0.795$ & & C-statistic $=0.806$ & & C-statistic $=0.812$ & \\
\hline \multicolumn{7}{|l|}{ All-Cause Mortality } \\
\hline PCP-HF Risk Score $\neq$ & $1.75(1.67,1.84)$ & $<0.0001$ & $1.42(1.35,1.50)$ & $<0.0001$ & $1.38(1.32,1.46)$ & $<0.0001$ \\
\hline (per doubling) & C-statistic $=0.750$ & & C-statistic $=0.765$ & & C-statistic $=0.770$ & \\
\hline
\end{tabular}

$P C P-H F$ pooled cohort equations to prevent heart failure

* $=$ Adjusted for age, sex, and race

$\dagger=$ Adjusted for age, sex, race, living status, living environment, income level, educational attainment, and previous history of myocardial infarction

$\neq=\log _{2}$ transformed

all-cause mortality $(\mathrm{C}$-statistic $=0.770)$. The associations of all-cause and CVD-mortality with quartiles of the PCP-HF risk score were consistent with results using the linear, continuous PCP-HF risk score (Supplemental Table 3). In fully adjusted models, individuals in the highest quartile of PCP-HF risk score had more than a 9-fold higher risk of CVD mortality compared to individuals in the lowest quartile of predicted 10-year HF risk $(\mathrm{HR}=9.71,95 \% \mathrm{CI}: 5.97,15.81 ; \mathrm{p}<0.0001)$. Allcause and CVD-mortality discrimination were similar for models including the continuous and categorized PCP-HF risk score. Neither sex nor race significantly modified the association of the PCP-HF risk score with CVD or all-cause mortality.

\section{Discussion}

This is the first study to characterize the 10-year risk for incident $\mathrm{HF}$ and assess the association of the recently derived PCP-HF risk score with CVD and all-cause mortality in a nationally representative sample of black and white men and women living in the United States. We found that the 10-year risk for incident HF varied according to social determinants of health, and groups with the highest HF risk included those with an annual household income below the poverty line, those living alone, as well as black participants. For each doubling of the PCP-HF risk score, individuals experienced a 58\% higher hazard of CVD-associated death, suggesting that increases in HF risk, even among those at a low overall risk, are clinically significant for prevention purposes. We observed a similar, albeit smaller risk of all-cause death associated with the PCP-HF risk score.

We sought to evaluate differences in predicted HF risk across key sociodemographic strata. While both predicted and actual CVD events vary by education and living status, income level, and living environment $[26,27]$, the role of these sociodemographic factors in predicting incident HF remains largely unexplored. Low socioeconomic status, and its correlates, including low educational attainment, are independent risk factors for CVD events, including non-fatal myocardial infarction and sudden cardiac death, in both men and women [28]. Given that myocardial infarction is the primary cause of HF worldwide [29], and CVD remains the leading cause of death globally, it is likely that poverty and environmental factors, collectively known as social determinants of health, similarly influence the risk for incident HF. Pathways that may help explain these associations include lower access to quality care and a higher burden of smoking, obesity, and physical inactivity among individuals of lower socioeconomic status [30]. While the PCP-HF risk score is perhaps the most complete and generalizable HF risk score, changing demographic trends and the increasing income inequality gap [31] in the United States suggest that even this new 2019 score may not fully capture ethnic and racial diversity in HF risk prediction.

These data underscore the expanded utility of the PCP-HF risk score as a valuable epidemiologic and clinical risk-stratification tool to prevent $\mathrm{HF}$ and, more broadly, downstream CVD mortality in the United States. While there are similar components between the PCP-HF and other HF risk scores, the PCP-HF is the first HF risk score to be validated among a sample including young adults and a high proportion of black men and women. Likewise, the PCP-HF robustly predicts HF incidence using data readily available in the primary care setting. This is in contrast to other HF risk scores $[9,11]$ that include more complex biological variables, including $\mathrm{N}$-terminal pro B-type natriuretic peptide, that are usually obtained more further downstream on the clinical HF workup. Our study lays the foundation for future endeavors that compare the PCP-HF risk score with current general CVD risk scores to assess whether patient motivation and primary prevention change based on the estimated risk across different models, even though the scores predict different clinical outcomes. For example, a patient at intermediate CVD risk may be more motivated by a $25-30 \%$ predicted risk of incident $\mathrm{HF}$ versus a $15 \%$ predicted risk of an CVD event over the next 10 years. 
Our study builds on previous reports regarding differences in HF incidence and risk factors. Analyses from the Atherosclerosis Risk in Communities (ARIC) study demonstrate that black males had the highest HF incidence, 9.1 cases per 1000 person-years, compared to black women, white men, and white women [32]. Likewise, the Coronary Artery Risk Development in Young Adults (CARDIA) study reported that black adults under the age of 50 years have a 20 -fold higher incidence of early onset HF, compared to whites [33]. Consistent with these observations, we found that black men had the highest predicted 10-year risk for incident $\mathrm{HF}$, at $2.3 \%$, followed by black women, $2.1 \%$, white men, $2.1 \%$, and white women, $1.1 \%$. These findings may be partially explained by considering the various different forms of HF. HF is a heterogenous disease with several different risk factors and disease subtypes, including heart failure with reduced ejection fraction (HFrEF) as well as heart failure with preserved ejection fraction (HFpEF). The lifetime risk of HFrEF is higher in men compared to women, while the lifetime risk of HFpEF is higher in whites versus blacks [34]. Furthermore, female sex is an established etiological risk factor for HFpEF [35].

In addition to documenting a higher risk of death due to CVD and all causes across increasing PCP-HF risk, we also identified discrete score cut offs that that may be used by clinicians to improve HF risk stratification. Similar to the Pooled Cohort Equations CVD risk score [36], there may low-, intermediate-, and high-risk zones for HF that could potentially guide clinical decision making. Based on observed CVD mortality rates across PCP-HF risk quartiles, a measured risk falling within quartiles 1 and 2 (0 to 2.7\%) appears to confer a low risk for a future CVD-attributed death, or less than 7 events per 1000 person years. On the other hand, calculated PCP-HF risk scores between 2.7 and 6.7\%, quartile 3, and greater than $6.7 \%$, quartile 4 , were associated with an intermediate- (15 events per 1000 person years) and high-risk (32 events per 1000 person years) for CVD mortality. Future studies are warranted to confirm our findings and improve the accuracy and definition of clinically relevant PCP-HF risk score categories.

Increases in population diversity, aging of the baby boomer population, and a rapidly increasing HF epidemic [1-3] have warranted urgent public health action to improve HF prevention. Our study, using a nationally representative sample of black and white men and women across different sociodemographic subgroups, serves to inform such strategies. Thus, utilization of NHANES data and HF risk estimation through sex- and race-specific equations enhances the generalizability of our findings and highlights the notable lack of race/ethnic-specific HF risk prediction tools available.
Other important strengths of this study include long follow-up time as well as the use of hard endpoints, including death due to CVD or all causes. On the other hand, we were not able to differentiate the association of the PCP-HF risk score with specific types of CVD-death (ex: HF, acute myocardial infarction, hypertensive heart disease, arrhythmia, etc.) due to the lack of disaggregated cause of death data. Availability of such data would have enabled us to further explore the discriminative ability of the PCP-HF risk score specifically associated with HF mortality. Another important consideration is that the PCP-HF risk score does not distinguish between predicted 10-year incidence of HFrEF versus HFpEF. This latter limitation is an essential factor to address in future studies given the changing epidemiological landscape of $\mathrm{HF}$, as the prevalence of HFpEF is growing and now accounts for at least one-half of all HF cases [37].

\section{Conclusions}

Here, we have identified a strong association between the PCP-HF risk score and CVD and all-cause mortality in a large, representative sample of United States adults. Individuals with the highest 10-year predicted risk for incident HF include those who reported a previous history of myocardial infarction, black participants, as well as persons with low educational attainment or an annual household income below $\$ 25,000$. Thus, social determinants of health may influence predicted HF risk and mortality, and future predictive equations should strive to capture these measures. Most importantly, these findings highlight the expanded utility of the PCP-HF risk score and suggest that its more formal use at both the clinical and population health levels may help improve primary CVD prevention in the United States.

\section{Supplementary information}

Supplementary information accompanies this paper at https://doi.org/10. 1186/s12872-020-01485-2.

Additional file 1: Table S1. Sex- and race-specific equation parameters for estimation of 10-year HF risk. Table S2. Person-Time and Crude Number of Deaths According to PCP-HF Risk Score Quartile. Table S3. Hazard of Cardiovascular Disease and All-Cause Mortality According to PCP-HF Risk Score Quartiles.

\section{Abbreviations}

BMI: Body mass index; CVD: Cardiovascular disease; lipoprotein; HF: Heart failure; HFpEF: Heart failure with preserved ejection fraction; HFrEF: Heart failure with reduced ejection fraction;

NHANES: National health and nutritional examination survey; PCP-HF: Pooled cohort equations to prevent heart failure

\section{Acknowledgements}

We would like to thank all participants and investigators of the National Health and Nutrition Examination Survey. We would like to also thank the National Center for Health Statistics. 


\section{Authors' contributions}

$A C R, C F$, and $L A B$ designed the work. ACR analyzed the data. ACR, KSP, TNK, $C F$, and $L A B$ interpreted the data. $A C R$ and $L A B$ drafted the work. ACR, KSP, TNK, JH, CF, MKW, AHA, JB, SPW, RSB, DLJ, and LAB substantively revised the work. All authors have read and approved the manuscript.

\section{Funding}

All research was conducted independently from funding agencies. Funding agencies had no role in the research design, data collection, analysis, interpretation, or composition of the current study and manuscript. Alexander Razavi is currently funded through a fellowship training grant supported by the National Heart, Lung, and Blood Institute of the National Institutes of Health under grant number F30HL 147486. Lydia Bazzano was supported in part by the National Institutes of Health under grant numbers: K12HD043451, P20GM109036, R21AG057983, R01HL121230 and R01DK091718. Tanika Kelly was supported in part by the National Institutes of Health under grant numbers: P20GM109036 and R21AG051914. 5R01AG041200-05. Jiang He was supported in part by the National Institutes of Health under grant number: P20GM109036. Marie Krousel-Wood was supported in part by the National Institutes of Health under grant numbers: K12HD043451, P20GM109036, UL1TR001417, U54GM104940; Amanda Anderson was supported in part by the National Institutes of Health under grant numbers: P20GM109036, R01DK104730, and R01DK107566.

\section{Availability of data and materials}

All data used for the current manuscript is online and currently available through NHANES III datasets (https://wwwn.cdc.gov/nchs/nhanes/nhanes3/ DataFiles.aspx) and the survey's linked mortality dataset (https://www.cdc.gov/ nchs/data-linkage/mortality.htm). This study did not use restricted access data.

\section{Ethics approval and consent to participate}

All individuals participating in NHANES III signed consent forms. Participants consented to storing blood specimens and clinical data for future research. All data and protocols in NHANES III were approved by the Centers for Disease Control and Prevention/National Center for Health Statistics ethics review board.

\section{Consent for publication}

Not applicable.

\section{Competing interests}

The authors declare that they have no competing interests.

\section{Author details}

'Department of Epidemiology, Tulane University School of Public Health and Tropical Medicine, 1440 Canal Street, Suite 2000, New Orleans, Louisiana 70112, USA. ${ }^{2}$ Department of Medicine, Tulane University School of Medicine, New Orleans, Louisiana, USA. ${ }^{3}$ The Ciccarone Center for the Prevention of Cardiovascular Disease, Johns Hopkins University School of Medicine, Baltimore, MD, USA. ${ }^{4}$ Department of Preventive Medicine, Northwestern University Feinberg School of Medicine, Chicago, IL, USA.

Received: 1 February 2020 Accepted: 14 April 2020 Published online: 25 April 2020

\section{References}

1. Heidenreich PA, Trogdon JG, Khavjou OA, et al. Forecasting the future of cardiovascular disease in the United States: a policy statement from the American Heart Association. Circulation. 2011. https://doi.org/10.1161/CIR. Ob013e31820a55f5

2. Vigen R, Maddox TM, Allen LA. Aging of the United States population: impact on heart failure. Curr Heart Fail Rep. 2012. https://doi.org/10.1007/ s11897-012-0114-8.

3. Savarese G, Lund LH. Global Public Health Burden of Heart Failure. Card Fail Rev. 2017. https://doi.org/10.15420/cfr.2016:25:2.

4. Sidney S, Go AS, Jaffe MG, Solomon MD, Ambrosy AP, Rana JS. Association between aging of the US population and heart disease mortality from 2011 to 2017. JAMA Cardiol. 2019. https://doi.org/10.1001/jamacardio.2019.4187.

5. Shah KS, Xu H, Matsouaka RA, et al. Heart failure with preserved, borderline, and reduced ejection fraction: 5-year outcomes. J Am Coll Cardiol. 2017; 70(20):2476-86. https://doi.org/10.1016/j.jacc.2017.08.074.
6. Vivo RP, Krim SR, Liang L, et al. Short- and long-term rehospitalization and mortality for heart failure in 4 racial/ethnic populations. J Am Heart Assoc. 2014;3(5). https://doi.org/10.1161/JAHA.114.001134.

7. He J, Ogden LG, Bazzano LA, Vupputuri S, Loria C, Whelton PK. Risk factors for congestive heart failure in US men and women: NHANES I epidemiologic follow-up study. Arch Intern Med. 2001;161(7):996-1002 http://www.ncbi.nlm.nih.gov/pubmed/11295963 Accessed 9 July 2019.

8. Khan SS, Ning H, Shah SJ, et al. 10-year risk equations for incident heart failure in the general population. J Am Coll Cardiol. 2019;73(19):2388-97. https://doi.org/10.1016/j.jacc.2019.02.057.

9. Agarwal SK, Chambless LE, Ballantyne CM, et al. Prediction of incident heart failure in general practice the atherosclerosis risk in communities (ARIC) study. Circ Hear Fail. 2012;5(4):422-9. https://doi.org/10.1161/ CIRCHEARTFAILURE.111.964841

10. Butler B, Kalogeropoulos A, Georgiopoulou V, et al. Incident heart failure prediction in the elderly: the health $A B C$ heart failure score. Circ Heart Fail. 2008;1(2):125-33. https://doi.org/10.1161/CIRCHEARTFAILURE. 108.768457

11. Chahal $\mathrm{H}$, Bluemke DA, Wu CO, et al. Heart failure risk prediction in the multi-ethnic study of atherosclerosis. Heart. 2015;101(1):58-64. https://doi. org/10.1136/heartjnl-2014-305697.

12. Kannel WB, D'Agostino RB, Silbershatz H, Belanger AJ, Wilson PWF, Levy D. Profile for estimating risk of heart failure. Arch Intern Med. 1999;159(11): 1197-204. https://doi.org/10.1001/archinte.159.11.1197.

13. Ho JE, Gona P, Pencina MJ, et al. Discriminating clinical features of heart failure with preserved vs. reduced ejection fraction in the community. Eur Heart J. 2012;33(14):1734-41. https://doi.org/10.1093/eurheartj/ehs070.

14. Solomon SD, McMurray JJV, Anand IS, et al. Angiotensin-Neprilysin inhibition in heart failure with preserved ejection fraction. N Engl J Med. 2019:381(17):1609-20. https://doi.org/10.1056/NEJMoa1908655.

15. Plan and operation of the Third National Health and Nutrition Examination Survey, 1988-94. Series 1: programs and collection procedures. Vital Health Stat 1. 1994;(32):1-407. http://www.ncbi.nlm.nih.gov/pubmed/7975354. Accessed 9 July 2019

16. National Center for Health Statistics. Public-use Linked Mortality File, 2015. 2015. https://www.cdc.gov/nchs/data/datalinkage/LMF2015_Methodology_ Analytic_Consider. Accessed 9 July 2019.

17. National Health and Nutrition Examination Survey III. Blood pressure quality control program. Rockville; 1989. https://wwwn.cdc.gov/nchs/data/nhanes3/ manuals/bpqc.pdf. Accessed 9 July 2019.

18. National Health and Nutrition Examination Survey III: Electrocardiogram. Rockville, MD; 1991. https://wwwn.cdc.gov/nchs/data/nhanes3/manuals/ecg. pdf. Accessed 9 July 2019

19. NATIONAL HEALTH AND NUTRITION EXAMINATION SURVEY III Body Measurements (Anthropometry).; 1988. https://wwwn.cdc.gov/nchs/data/ nhanes3/manuals/anthro.pdf. Accessed 9 July 2019.

20. Gunter EW, Lewis BG, Koncikowski SM. Laboratory procedures used for the third National Health and Nutrition Examination Survey (NHANES III), 19881994. https://wwwn.cdc.gov/nchs/data/nhanes3/manuals/labman.pdf. Accessed 9 July 2019.

21. Center for Health Statistics N. The Third National Health and Nutrition Examination Survey (NHANES III) Linked Mortality File: Mortality Follow-up through 2006. http://www.cdc.gov/nchs/data/datalinkage/matching methodology_nhanes3_final.pdf. Accessed 9 July 2019.

22. Keyfitz N. Sampling variance of standardized mortality rates. Hum Biol. 1966:38(3): 309-17. http://www.ncbi.nlm.nih.gov/pubmed/5977534. Accessed July 18, 2019.

23. Havranek EP, Mujahid MS, Barr DA, et al. Social determinants of risk and outcomes for cardiovascular disease. Circulation. 2015;132(9):873-98. https:// doi.org/10.1161/CIR.0000000000000228.

24. Pencina MJ, D'Agostino RB. Evaluating discrimination of risk prediction models: the C statistic. JAMA - J Am Med Assoc. 2015. https://doi.org/10. 1001/jama.2015.11082

25. Uno H, Cai T, Pencina MJ, D'Agostino RB, Wei LJ. On the C-statistics for evaluating overall adequacy of risk prediction procedures with censored survival data. Stat Med. 2011. https://doi.org/10.1002/sim.4154.

26. Colantonio LD, Richman JS, Carson AP, et al. Performance of the atherosclerotic cardiovascular disease pooled cohort risk equations by social deprivation status. J Am Heart Assoc. 2017;6(3). https://doi.org/10.1161/ JAHA.117.005676.

27. Rana JS, Tabada GH, Solomon MD, et al. Accuracy of the atherosclerotic cardiovascular risk equation in a large contemporary, Multiethnic 
Population.; 2016. http://www.onlinejacc.org/content/accj/67/18/2118.full. pdf. Accessed 9 July 2019.

28. Kucharska-Newton AM, Harald K, Rosamond WD, Rose KM, Rea TD, Salomaa $V$. Socioeconomic indicators and the risk of acute coronary heart disease events: comparison of population-based data from the United States and Finland. Ann Epidemiol. 2011;21(8):572-9. https://doi.org/10.1016/j. annepidem.2011.04.006.

29. Cahill TJ, Kharbanda RK. Heart failure after myocardial infarction in the era of primary percutaneous coronary intervention: mechanisms, incidence and identification of patients at risk. World J Cardiol. 2017;9(5):407. https://doi. org/10.4330/wjc.v9.15.407.

30. Kershaw KN, Droomers M, Robinson WR, Carnethon MR, Daviglus ML, Monique Verschuren WM. Quantifying the contributions of behavioral and biological risk factors to socioeconomic disparities in coronary heart disease incidence: the MORGEN study. Eur J Epidemiol. 2013;28(10):807-14. https:// doi.org/10.1007/s10654-013-9847-2.

31. Suro Ri, Passel JS, 10p. The Rise of the Second Generation: Changing Patterns in Hispanic Population Growth.; 2003. http://www.pewhispanic.org. Accessed 21 Nov 2019.

32. Loehr LR, Rosamond WD, Chang PP, Folsom AR, Chambless LE. Heart failure incidence and survival (from the atherosclerosis risk in communities study). Am J Cardiol. 2008;101(7):1016-22. https://doi.org/10.1016/.amjcard.2007.11.061.

33. Bibbins-Domingo K, Pletcher MJ, Lin F, et al. Racial differences in incident heart failure among young adults. N Engl J Med. 2009;360(12):1179-90. https://doi.org/10.1056/NEJMoa0807265.

34. Pandey A, Omar W, Ayers C, et al. Sex and race differences in lifetime risk of heart failure with preserved ejection fraction and heart failure with reduced ejection fraction. Circulation. 2018;137(17):1814-23. https://doi.org/10.1161/ CIRCULATIONAHA.117.031622.

35. Bursi F, Weston SA, Redfield MM, et al. Systolic and diastolic heart failure in the community. JAMA. 2006;296(18):2209-16. https://doi.org/10.1001/jama. 296.18.2209.

36. Arnett DK, Blumenthal RS, Albert MA, et al. 2019 ACC/AHA guideline on the primary prevention of cardiovascular disease. Circulation. March 2019. https://doi.org/10.1161/CIR.0000000000000678.

37. Dunlay SM, Roger VL, Redfield MM. Epidemiology of heart failure with preserved ejection fraction. Nat Rev Cardiol. 2017. https://doi.org/10.1038/ nrcardio.2017.65

\section{Publisher's Note}

Springer Nature remains neutral with regard to jurisdictional claims in published maps and institutional affiliations.

Ready to submit your research? Choose BMC and benefit from:

- fast, convenient online submission

- thorough peer review by experienced researchers in your field

- rapid publication on acceptance

- support for research data, including large and complex data types

- gold Open Access which fosters wider collaboration and increased citations

- maximum visibility for your research: over $100 \mathrm{M}$ website views per year

At $\mathrm{BMC}$, research is always in progress.

Learn more biomedcentral.com/submissions 